\title{
Lipoprotein Ratios as Surrogate Markers for Insulin Resistance in South Indians with Normoglycemic Nondiabetic Acute Coronary Syndrome
}

\author{
Medha Rajappa, ${ }^{1}$ M. G. Sridhar, ${ }^{1}$ J. Balachander, ${ }^{2}$ \\ K. R. Sethuraman, ${ }^{3}$ and Kalai Selvi Rajendiran ${ }^{1}$ \\ ${ }^{1}$ Department of Biochemistry, Jawaharlal Institute of Postgraduate Medical Education and Research, Puducherry 605 006, India \\ ${ }^{2}$ Department of Cardiology, Jawaharlal Institute of Postgraduate Medical Education and Research, Puducherry 605 006, India \\ ${ }^{3}$ Department of Medicine, Jawaharlal Institute of Postgraduate Medical Education and Research, Puducherry 605 006, India
}

Correspondence should be addressed to M. G. Sridhar; sridhar_biochem@yahoo.co.in

Received 20 February 2014; Accepted 8 April 2014; Published 18 May 2014

Academic Editors: C. Bizzarri, Y. Combarnous, M. Karadeniz, and N. Tentolouris

Copyright (C) 2014 Medha Rajappa et al. This is an open access article distributed under the Creative Commons Attribution License, which permits unrestricted use, distribution, and reproduction in any medium, provided the original work is properly cited.

\begin{abstract}
Background. Insulin resistance has been associated with dyslipidemia and cardiovascular disease. Even though homeostasis model assessment of insulin resistance (HOMA-IR) is a well-known insulin resistance predictor, estimation of serum lipoprotein ratios has been recently suggested as a surrogate marker for insulin resistance. Here, we evaluated the relationship between lipoprotein ratios and insulin resistance in normoglycemic nondiabetic south Indians with acute coronary syndrome. Methods. 100 normoglycemic nondiabetic ACS patients and 140 controls were enrolled in the study. Levels of fasting glucose, fasting insulin, and lipid profile [total cholesterol (TC), triglycerides (TG), and high density lipoprotein cholesterol (HDL-C)], lipoprotein(a) [Lp(a)] levels were measured and lipoprotein ratios were computed. HOMA-IR was used to calculate the insulin resistance. Receiver operating characteristic curves (ROC) analysis was used to compare the power of these lipoprotein ratios to predict insulin resistance. Results. Lipoprotein ratios were significantly higher in normoglycemic nondiabetic ACS patients, as compared to healthy controls, and were significantly correlated with HOMA-IR by Spearman's rank correlation analysis. ROC curve showed that Lp(a)/HDL-C and TG/HDL-C ratios were the best surrogate predictors of insulin resistance in normoglycemic nondiabetic ACS. Conclusion. This study demonstrates that serum lipoprotein ratios significantly correlate with insulin resistance in normoglycemic nondiabetic ACS. $\mathrm{Lp}(\mathrm{a}) / \mathrm{HDL}-\mathrm{C}$ and TG/HDL-C ratios could be used as surrogate markers of insulin resistance in atherosclerosis-prone south Indians with normoglycemic nondiabetic ACS.
\end{abstract}

\section{Introduction}

Coronary artery disease (CAD) has been reported as the first leading cause of death in South Asia, accounting for $13.6 \%$ of all deaths [1]. Also, there are extreme severity and prematurity of acute coronary syndromes (ACS) in Asian Indians who were found to have high mortality from this disease specifically from the Indian subcontinent [2]. Insulin resistance (IR) has been suggested as a risk factor for developing ACS [3]. Lee et al. have reported that despite insulin resistance being associated with conventional risk factors, it plays a role in the evolution of coronary atherosclerotic plaques in asymptomatic subjects [4]. Insulin resistance seems to be a significant risk factor for coronary events [5]. Plasma triglyceride (TG) level, independently associated with insulin resistance and hyperinsulinemia, is an independent predictor of CAD [6]. A previous study has shown that TG/HDL-C ratio is a significant predictor of cardiovascular disease [7]. Lipoprotein(a) $[\mathrm{Lp}(\mathrm{a})]$ also correlates with coronary artery disease with insulin resistance such as type 2 diabetes mellitus [8].

The use of simple ratios to identify insulin resistance would be of immense clinical use, as surrogate markers for insulin resistance. The current methods to evaluate insulin resistance involving sophisticated methodology are expensive and time-consuming. Hence, there is an urgent 
TABLE 1: Baseline characteristics of patients with ACS and controls.

\begin{tabular}{|c|c|c|c|}
\hline Parameters & $\begin{array}{c}\text { Cases }(n=100) \\
\text { mean } \pm \text { SD }\end{array}$ & $\begin{array}{c}\text { Controls }(n=140) \\
\text { mean } \pm \text { SD }\end{array}$ & $P$ value \\
\hline Age (years) & $51.03 \pm 12.59$ & $51.16 \pm 11.85$ & 0.422 \\
\hline Gender (male : female) & $85: 15$ & $111: 29$ & - \\
\hline $\mathrm{BMI}\left(\mathrm{kg} / \mathrm{m}^{2}\right)$ & $25.83 \pm 5.61$ & $21.83 \pm 2.30$ & $<0.01$ \\
\hline $\mathrm{W} / \mathrm{H}$ ratio & $0.942 \pm 0.06$ & $0.938 \pm 0.06$ & 0.446 \\
\hline Systolic BP (mm Hg) & $130.86 \pm 16.72$ & $114.39 \pm 9.2$ & $<0.01$ \\
\hline Diastolic BP (mm Hg) & $85.40 \pm 13.65$ & $75.02 \pm 7.7$ & $<0.01$ \\
\hline Unstable angina (\%) & 31 & - & - \\
\hline Non-Q wave MI (\%) & 2 & - & - \\
\hline Q-wave acute MI (\%) & 67 & - & - \\
\hline
\end{tabular}

need to develop a relatively simple and economic method to investigate insulin resistance in ACS. We aimed to explore the utility of simple lipoprotein ratios as surrogate markers in the diagnosis of insulin resistance in patients with normoglycemic nondiabetic patients with ACS.

\section{Methods}

This hospital-based case-control study included 100 normoglycemic nondiabetic ACS patients presenting to the medical intensive care unit (MICU) of the hospital, within 12 hours of onset of symptoms, with or without ECG changes, and 140 healthy controls. The study protocol was approved by the Institute of Ethics Committee (Human Studies). Acute coronary syndrome was diagnosed based on clinical, electrocardiographic, and biochemical criteria. The patients were enrolled in the study group after giving informed consent and filling in a structured questionnaire, including details of classical risk factors such as family history of CAD, hypertension, and smoking. Also enrolled, after giving informed consent, were 140 nondiabetic, healthy controls who satisfied the following criteria: normal glucose tolerance test, absence of angina (Rose questionnaire), absence of history of any vascular disease [acute myocardial infarction (AMI), stroke, or intermittent claudication], normal 12-lead resting electrocardiograms, and no past history of any cardiac illness, hypertension, diabetes mellitus, and other metabolic illnesses.

The present study did not include patients or control subjects with a history of endocrine, metabolic, neoplastic, hepatic, renal, infectious, autoimmune, or peripheral arterial disease, pregnancy, and surgical correction in preceding 6 months or those on any medication such as antiinflammatory or lipid lowering drugs. After written informed consent was obtained from all study subjects, a fasting blood sample was taken within 12 hours of presentation to the hospital and plasma was separated.

Plasma glucose, total cholesterol, triglyceride and HDLcholesterol (after LDL precipitation with heparin- $\mathrm{MnCl}_{2}$ ) were estimated on the same day using commercial kits on the 550 Express autoanalyzer. The intra-assay and interassay CVs were $4 \%$ to $6 \%$, within the recommended range suggested by the National Cholesterol Education Program [9].
LDL-cholesterol was calculated using Friedewald's formula [10]. The remaining plasma was stored at $-80^{\circ} \mathrm{C}$, until the assays were performed. Lp (a) was determined quantitatively by "INNOTEST Lp(a) ELISA" (Innogenetics NV, Belgium). Insulin was determined by radioimmunoassay (RIA) (radiopharmaceuticals and labelled compounds, Board of Radiation and Isotope Technology, BARC, Vashi Complex, NAVI, Mumbai, India). Insulin resistance indices were calculated by the formula: HOMA IR $=[$ fasting glucose $(\mathrm{mg} / \mathrm{dL}) \times$ fasting insulin $(\mu \mathrm{IU} / \mathrm{mL})] / 405$ [11]. Patients with HOMA-IR $\geq 2$ were defined as insulin resistant. Patients with HOMA-IR $<2$ were classified as insulin sensitive.

\section{Statistical Analysis}

Baseline characteristics of cases and controls were analyzed using descriptive statistics. The normality of continuous data was assessed by Kolmogorov-Smirnov test. The data were described as mean \pm standard deviation and compared by Mann-Whitney $U$ test. Association of risk factors between the two groups was assessed by chi-square test. Correlation between biochemical parameters was studied using Spearman's rank correlation test. To determine which lipoprotein ratio best predicts the insulin resistance (HOMA $>2$ ), a receiver operating characteristic curve (ROC) was constructed and the area under the curve was calculated. Analysis was carried out at 5\% level of significance and $P<0.05$ was considered as statistically significant. Statistical analysis was performed using IBM SPSS statistics version 20 for windows.

\section{Results}

Table 1 showed the baseline characteristics of the study groups. BMI and systolic and diastolic BP were significantly higher in the ACS patients, as compared with controls. $67 \%$ of patients had Q-wave AMI, 31\% had unstable angina, and $2 \%$ had non-Q wave AMI.

Table 2 shows the association of risk factors in nondiabetic ACS patients and controls. The risk factors were significantly higher in patients with nondiabetic ACS, as compared with controls. 
TABLE 2: Association of risk factors between patients of nondiabetic ACS $(n=100)$ and controls $(n=140)$ by chi-square test.

\begin{tabular}{|c|c|c|c|c|}
\hline Risk factors & Cases $n(\%)$ & Controls $n(\%)$ & $\chi^{2}$ value & $P$ value \\
\hline Smoking & $72(72)$ & $29(20.71)$ & 62.95 & $<0.01$ \\
\hline Hypertension & $29(29)$ & - & 46.18 & $<0.01$ \\
\hline LVH & $12(12)$ & - & 19.242 & $<0.01$ \\
\hline Obesity & $51(51)$ & $8(5.7)$ & 64.52 & $<0.01$ \\
\hline Postmenopausal & $15(15)$ & $19(13.57)$ & 7.46 & 0.024 \\
\hline High TG & $37(37)$ & - & 61.241 & $<0.01$ \\
\hline High LDL cholesterol & $62(62)$ & - & 117.0 & $<0.01$ \\
\hline High total cholesterol & $51(51)$ & - & 90.67 & $<0.01$ \\
\hline Low HDL cholesterol & $62(62)$ & - & 117.0 & $<0.01$ \\
\hline Alcoholism & $24(24)$ & $5(3.57)$ & 22.92 & $<0.01$ \\
\hline Family history of CAD & $39(39)$ & - & 65.14 & $<0.01$ \\
\hline Past history of CAD & $19(19)$ & - & 28.88 & $<0.01$ \\
\hline
\end{tabular}

TABLE 3: Comparison of biochemical parameters between nondiabetic ACS and controls.

\begin{tabular}{lccc}
\hline Parameters & $\begin{array}{c}\text { Cases }(n=100) \\
\text { mean } \pm \text { SD }\end{array}$ & $\begin{array}{c}\text { Controls }(n=140) \\
\text { mean } \pm \text { SD }\end{array}$ & $\begin{array}{c}P \text { value } \\
(\text { Mann-Whitney } U \text { test })\end{array}$ \\
\hline Fasting glucose $(\mathrm{mg} / \mathrm{dL})$ & $76.8 \pm 5.5$ & $66.1 \pm 7.0$ & 0.034 \\
Insulin $(\mu \mathrm{IU} / \mathrm{mL})$ & $19.9 \pm 3.7$ & $13.5 \pm 2.7$ & 0.012 \\
HOMA-IR & $3.8 \pm 1.3$ & $2.6 \pm 0.7$ & 0.033 \\
Total cholesterol (mg/dL) & $225.2 \pm 40.1$ & $155.4 \pm 26.0$ & $<0.001$ \\
LDL cholesterol (mg/dL) & $149.0 \pm 33.1$ & $88.0 \pm 23.50$ & $<0.001$ \\
VLDL cholesterol (mg/dL) & $32.7 \pm 10.7$ & $16.4 \pm 6.0$ & $<0.001$ \\
HDL cholesterol $(\mathrm{mg} / \mathrm{dL})$ & $43.9 \pm 16.09$ & $50.87 \pm 10.29$ & $<0.001$ \\
TG $(\mathrm{mg} / \mathrm{dL})$ & $162.3 \pm 46.1$ & $82.23 \pm 28.5$ & $<0.001$ \\
Lipoprotein(a) $(\mathrm{mg} / \mathrm{dL})$ & $68.2 \pm 22.2$ & $17.9 \pm 6.20$ & $<0.001$ \\
Total CK $(\mathrm{IU} / \mathrm{L})$ & $631.3 \pm 648.9$ & $56.6 \pm 28.9$ & $<0.01$ \\
CK-2/CK-MB $(\mathrm{IU} / \mathrm{L})$ & $77.70 \pm 64.5$ & $14.02 \pm 6.7$ & $<0.01$ \\
AST $(\mathrm{IU} / \mathrm{L})$ & $290.8 \pm 363.0$ & $31.7 \pm 34.1$ & $<0.01$ \\
\hline
\end{tabular}

Routine biochemical analytes are depicted in Table 3. Fasting glucose, fasting insulin, HOMA-IR, lipid parameters (other than HDL-c), lipoprotein(a) and cardiac enzymes, creatine kinase (CK), CK-2/CK-MB, and AST were significantly higher in nondiabetic ACS than in controls $(P<0.01)$.

Lipid indices such as non-HDL-C, TC/HDL-C, LDL-C/ HDL-C, TG/HDL-C, non-HDL-C/HDL-C, and Lp(a)/HDL$\mathrm{C}$ were significantly higher $(P<0.001)$ in nondiabetic ACS patients than in controls (Table 4). Correlation of all these lipoprotein ratios with HOMA-IR is shown in Table 5, where all lipoprotein ratios correlated significantly.

Figure 1 depicts the ROC curve for the lipid ratios to detect insulin resistance. Table 6 showed area under curve of the lipid ratios indices for prediction of insulin resistance in normo-glycemic non-diabetic ACS patients. Area under the curve was maximum for the two ratios, TG/HDL-C (0.933) and $\mathrm{Lp}(\mathrm{a}) / \mathrm{HDL}-\mathrm{C}(0.968)$, for prediction of insulin resistance in nondiabetic ACS. Among all lipid indices, Lp(a)/HDL-C was the best predictor and showed sensitivity of $96.6 \%$ and specificity of $82.9 \%$ with cut-off value of 0.295 . TG/HDL-C showed sensitivity of $88.8 \%$ and specificity of $82.9 \%$, with cutoff value of 1.463 , as shown in Table 7 .

\section{Discussion}

Our study population constituted the CAD-prone south Indian population, who showed a significant elevation in the HOMA-IR index in normoglycemic nondiabetic ACS patients when compared to the controls. This is in accordance with a previous study by Lazerri and coworkers in nondiabetic STEMI patients [12]. Association of insulin resistance with lipoprotein ratios has been shown in patients with type 2 diabetes mellitus [13].

Insulin resistance is characterized by not only decreased glucose utilization by tissues in response to insulin but also myriad of events that increase significantly the risk for cardiovascular disease [14]. The multifactorial pathogenesis of insulin resistance syndrome and associated atherogenic dyslipidemia is very complex. Metabolic dysregulation of fatty acids is at the heart of the pathophysiology of the insulin resistance syndrome [14].

Although HOMA-IR is gaining wide acceptance as a measure for insulin resistance, there is no consensus regarding its cut-off value for identification of insulin resistance [15]. This is true for Indian subjects also. In our study, insulin resistance 
TABLE 4: Comparison of lipid indices in study populations.

\begin{tabular}{lccc}
\hline Parameters & $\begin{array}{c}\text { Cases }(n=100) \\
\text { mean } \pm \text { SD }\end{array}$ & $\begin{array}{c}\text { Controls }(n=140) \\
\text { mean } \pm \text { SD }\end{array}$ & $\begin{array}{c}P \text { value } \\
(\text { Mann-Whitney } U \text { test })\end{array}$ \\
\hline Non-HDL-C (mg/dL) & $189.1 \pm 45.0$ & $104.5 \pm 28.5$ & $<0.001$ \\
TC/HDL-C & $7.0 \pm 3.9$ & $3.2 \pm 0.9$ & $<0.001$ \\
LDL/HDL-C & $4.7 \pm 3.0$ & $1.8 \pm 0.7$ & $<0.001$ \\
TG/HDL-C & $5.1 \pm 3.5$ & $1.7 \pm 0.7$ & $<0.001$ \\
Non-HDL-C/HDL-C & $6.0 \pm 3.9$ & $2.2 \pm 0.9$ & $<0.001$ \\
Lp(a)/HDL-C & $2.1 \pm 1.1$ & $0.4 \pm 0.1$ & $<0.001$ \\
\hline
\end{tabular}

TABLE 5: Correlation coefficients of lipid indices with HOMA-IR by Spearman's rank correlation analysis.

\begin{tabular}{lcc}
\hline Lipid indices & $r$ value & $P$ value \\
\hline Non-HDL-C & 0.635 & $<0.001$ \\
TC/HDL-C & 0.640 & $<0.001$ \\
LDL/HDL-C & 0.641 & $<0.001$ \\
TG/HDL-C & 0.789 & $<0.001$ \\
Non-HDL/HDL-C & 0.640 & $<0.001$ \\
Lp(a)/HDL-C & 0.868 & $<0.001$ \\
\hline
\end{tabular}

was identified as HOMA-IR $\geq 2$ and insulin sensitivity was identified by HOMA-IR $<2$. This was in line with earlier studies by Sinha et al. [16] and Ray et al. [17].

Several studies have demonstrated that the TC/HDL$\mathrm{C}$ and the LDL-C/HDL-C ratios are better predictors of atherosclerosis and cardiovascular disease than single lipid markers [18-21]. In our study, all the lipid ratios were elevated in the group of normoglycemic nondiabetic ACS, as compared with controls, and correlated significantly with insulin resistance, as measured by HOMA-IR index.

In normoglycemic nondiabetic ACS patients who had HOMA-IR > 2, all lipoprotein ratios had significant ability of detecting insulin resistance shown by area under curve; however, the best predictors of insulin resistance were Lp(a)/HDL-C ratio, which showed sensitivity of $96.6 \%$ and specificity of $82.9 \%$ with cut-off value of 0.295 , and TG/HDL$\mathrm{C}$ ratio which showed sensitivity of $88.8 \%$ and specificity of $82.9 \%$, with cut-off value of 1.463 . In contrast to our findings, a study in the African American population showed that TG/HDL-C was not a reliable marker for insulin resistance [21].

$\mathrm{Lp}(\mathrm{a})$, an important genetic contributor in the progress of myocardial infarction and other cardiovascular diseases, has been shown to correlate with clinically expressed ACS [22]. In our study, Lp(a)/HDL-C ratio was the best predictor and surrogate marker for insulin resistance in normoglycemic nondiabetic ACS patients.

A recent study by Ray et al. has showed TG/HDL-C and TC/HDL-C as insulin resistance markers in the ACS patients with impaired fasting glucose [17]. Our study included ACS patients who had normal glycemic levels and we also included Lp(a), along with the lipid profile parameters in the lipid ratios for prediction of insulin resistance, as south Indians are genetically known to have higher preponderance for CAD

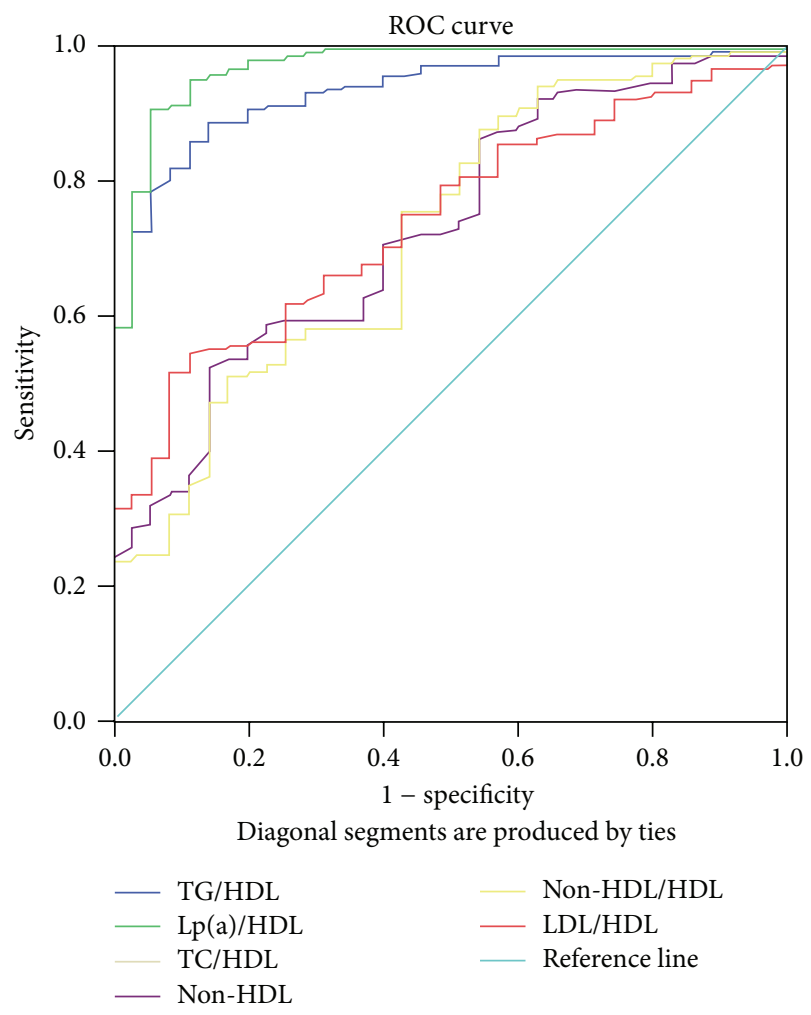

FIgURE 1: ROC curve for the lipid indices for the insulin resistance prediction.

and higher Lp(a) levels and high incidence of central obesity and insulin resistance [23-27].

Insulin resistance syndrome, predominantly found in Indians, is associated with increased atherothrombosis [12]. Insulin resistance syndrome produces a prothrombotic state due to the stimulatory effects of insulin on smooth muscle proliferation and migration of smooth muscle from media to intima and the increased levels of platelet activator inhibitor-1 (PAI-1) [14]. Lp(a) also contributes to the disease progression in CAD as it leads to the upregulation of PAI-1, which is known to be elevated in insulin resistance states [8]. All these effects may be potentiated by concomitant dyslipidemias. This probably explains the highly significant positive correlation of $\mathrm{Lp}(\mathrm{a}) / \mathrm{HDL}$ ratio with insulin resistance in the present study. 
TABLE 6: Area under the ROC curve for the insulin resistance in nondiabetic ACS.

\begin{tabular}{lccr}
\hline Lipid indices & AUC \pm SE & $95 \%$ CI & $P$ value \\
\hline Non-HDL-C & $0.730 \pm 0.043$ & $(0.646-0.814)$ & $<0.001$ \\
TC/HDL-C & $0.723 \pm 0.046$ & $(0.633-0.813)$ & $<0.001$ \\
LDL/HDL-C & $0.741 \pm 0.038$ & $(0.667-0.815)$ & $<0.001$ \\
TG/HDL-C & $0.933 \pm 0.018$ & $(0.897-0.969)$ & $<0.001$ \\
Non-HDL-C/HDL-C & $0.723 \pm 0.046$ & $(0.633-0.813)$ & $<0.001$ \\
Lp(a)/HDL-C & $0.968 \pm 0.014$ & $(0.940-0.996)$ & $<0.001$ \\
\hline
\end{tabular}

SE: standard error; CI: confidence interval.

TABLE 7: Cut-off points corresponding to the highest percentage of sensitivity and specificity calculated from ROC curves for the detection of insulin resistance in nondiabetic ACS.

\begin{tabular}{lccc}
\hline Lipid indices & Cut-off points & Sensitivity (\%) & Specificity (\%) \\
\hline Lp(a)/HDL-C & 0.295 & 96.6 & 82.9 \\
TG/HDL-C & 1.463 & 88.8 & 82.9 \\
\hline
\end{tabular}

A major limitation of the present study was that we used HOMA-IR as an estimate of insulin resistance and not the "gold-standard" method hyperinsulinemic hypoglycemic clamp technique. If we had used this technique, it would have validated the findings further.

We conclude that the Lp(a)/HDL-C and TG/HDL-C ratios could be used as surrogate measures of insulin resistance in normoglycemic nondiabetic CAD-prone south Indians with ACS. Since $\mathrm{Lp}(\mathrm{a})$ is a nonmodifiable genetic risk factor, there is a need to create awareness for early detection and modification of other risk factors in young individuals, to prevent the early onset of insulin resistance and premature CAD in south Indian population.

\section{Conflict of Interests}

The authors declare that there is no conflict of interests regarding the publication of this paper.

\section{References}

[1] C. D. Mathers, J. A. Salomon, M. Ezzati, S. Begg, S. VanderHoorn, and A. D. Lopez, Global Burden of Disease and Risk Factors, Oxford University Press, New York, NY, USA, 2006.

[2] E. A. Enas, J. Dhawan, and S. Petkar, "Coronary artery disease in Asian Indians. Lessons learnt and the role of Lp(a)," Indian Heart Journal, vol. 47, pp. 25-34, 1997.

[3] S. E. Karakas, K. Kyoungmi, and A. J. Duleba, "Determinants of impaired fasting glucose versus glucose intolerance in polycystic ovary syndrome," Diabetes Care, vol. 33, no. 4, pp. 887-893, 2010.

[4] K. K. Lee, S. P. Fortmann, J. M. Fair et al., "Insulin resistance independently predicts the progression of coronary artery calcification," American Heart Journal, vol. 157, no. 5, pp. 939945, 2009.

[5] J. Jeppesen, F. S. Facchini, and G. M. Reaven, "Individuals with high total cholesterol/HDL cholesterol ratios are insulin resistant," Journal of Internal Medicine, vol. 243, no. 4, pp. 293298, 1998.
[6] J. E. Hokanson and M. A. Austin, "Plasma triglyceride level is a risk factor for cardiovascular disease independent of high-density lipoprotein cholesterol level: a meta-analysis of population-based prospective studies," Journal of Cardiovascular Risk, vol. 3, pp. 213-219, 1996.

[7] J. M. Gaziano, C. H. Hennekens, C. J. O’Donnell, J. L. Breslow, and J. E. Buring, "Fasting triglycerides, high-density lipoprotein, and risk of myocardial infarction," Circulation, vol. 96, pp. 2520-2525, 1997.

[8] G. F. Watts, R. M. Ap Gwilym, J. Mazurkiewicz, and J. Coltart, "Independent correlation between plasma lipoprotein(a) and angiographic coronary artery disease in NIDDM," Diabetes Care, vol. 18, no. 2, pp. 234-236, 1995.

[9] S. M. Grundy, "National cholesterol education program: second report of the expert panel on detection, evaluation, and treatment of high blood cholesterol in adults (adult treatment panel II)," Circulation, vol. 89, no. 3, pp. 1329-1443, 1994.

[10] W. T. Friedewald, R. I. Levy, and D. S. Fredrickson, "Estimation of the concentration of low-density lipoprotein cholesterol in plasma, without use of the preparative ultracentrifuge," Clinical Chemistry, vol. 18, no. 6, pp. 499-502, 1972.

[11] D. R. Matthews, J. P. Hosker, and A. S. Rudenski, "Homeostasis model assessment: insulin resistance and $\beta$-cell function from fasting plasma glucose and insulin concentrations in man," Diabetologia, vol. 28, no. 7, pp. 412-419, 1985.

[12] C. Lazzeri, S. Valente, M. Chiostri, C. Picariello, and G. F. Gensini, "Correlates of acute insulin resistance in the early phase of non-diabetic ST-elevation myocardial infarction," Diabetes and Vascular Disease Research, vol. 8, no. 1, pp. 35-42, 2011.

[13] S. Tangvarasittichai, P. Poonsub, and O. Tangvarasittichai, "Association of serum lipoprotein ratios with insulin resistance in type 2 diabetes mellitus," Indian Journal of Medical Research, vol. 131, no. 5, pp. 641-648, 2010.

[14] H. N. Ginsberg, "Insulin resistance and cardiovascular disease," Journal of Clinical Investigation, vol. 106, no. 4, pp. 453-458, 2000.

[15] Z. Radikova, J. Koska, M. Huckova et al., "Insulin sensitivity indices: a proposal of cut-off points for simple identification of insulin-resistant subjects," Experimental and Clinical Endocrinology and Diabetes, vol. 114, no. 5, pp. 249-256, 2006.

[16] D. P. Sinha, S. Ahmed, A. K. Baneerjee, M. Das, and H. Hassan, "Significance of an index of insulin resistance in non- diabetic patients with impaired fasting glucose with acute myocardial infarction and its correlation to short term outcome," Indian Heart Journal, vol. 61, no. 1, pp. 40-43, 2009.

[17] S. Ray, A. K. Bairagi, S. Guha et al., "A simple way to identify insulin resistance in non-diabetic acute coronary syndrome patients with impaired fasting glucose," Indian Journal of Endocrinology and Metabolism, vol. 16, pp. 460-464, 2012. 
[18] B. Kinosian, H. Glick, and G. Garland, "Cholesterol and coronary heart disease: predicting risk by levels and ratios," Annals of Internal Medicine, vol. 121, no. 9, pp. 641-647, 1994.

[19] M. J. Stampfer, F. M. Sacks, S. Salvini, W. C. Willett, and C. H. Hennekens, "A prospective study of cholesterol, apolipoproteins, and the risk of myocardial infarction," The New England Journal of Medicine, vol. 325, no. 6, pp. 373-381, 1991.

[20] P. M. Ridker, M. J. Stampfer, and N. Rifai, "Novel risk factors for systemic atherosclerosis: a comparison of C-reactive protein, fibrinogen, homocysteine, lipoprotein(a), and standard cholesterol screening as predictors of peripheral arterial disease," Journal of the American Medical Association, vol. 285, no. 19, pp. 2481-2485, 2001.

[21] A. E. Sumner, K. B. Finley, D. J. Genovese, M. H. Criqui, and R. C. Boston, "Fasting triglyceride and the triglyceride-HDL cholesterol ratio are not markers of insulin resistance in African Americans," Archives of Internal Medicine, vol. 165, no. 12, pp. 1395-1400, 2005.

[22] J.-J. Wang, C.-N. Zhang, Y. Meng, A.-Z. Han, J.-B. Gong, and K. Li, "Elevated concentrations of oxidized lipoprotein(a) are associated with the presence and severity of acute coronary syndromes," Clinica Chimica Acta, vol. 408, no. 1-2, pp. 79-82, 2009.

[23] M. Rajappa, M. G. Sridhar, J. Balachander, and K. R. Sethuraman, "Lipoprotein (a) and comprehensive lipid tetrad index as a marker for coronary artery disease in NIDDM patients in South India," Clinica Chimica Acta, vol. 372, no. 1-2, pp. 70-75, 2006.

[24] F. S. Geethanjali, K. Luthra, A. Lingenhel et al., "Analysis of the apo(a) size polymorphism in Asian Indian populations: association with Lp(a) concentration and coronary heart disease," Atherosclerosis, vol. 169, no. 1, pp. 121-130, 2003.

[25] R. C. Hoogeveen, J. K. Gambhir, D. S. Gambhir et al., "Evaluation of Lp[a] and other independent risk factors for CHD in Asian Indians and their USA counterparts," Journal of Lipid Research, vol. 42, no. 4, pp. 631-638, 2001.

[26] J. K. Gambhir, H. Kaur, D. S. Gambhir, and K. M. Prabhu, "Lipoprotein(a) as an independent risk factor for coronary artery disease in patients below 40 years of age," Indian Heart Journal, vol. 52, no. 4, pp. 411-415, 2000.

[27] J. K. Gambhir, H. Kaur, K. M. Prabhu, J. D. Morrisett, and D. S. Gambhir, "Association between lipoprotein(a) levels, apo(a) isoforms and family history of premature CAD in young Asian Indians," Clinical Biochemistry, vol. 41, no. 7-8, pp. 453-458, 2008. 


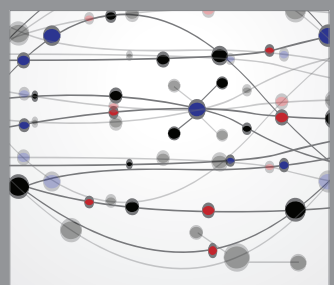

The Scientific World Journal
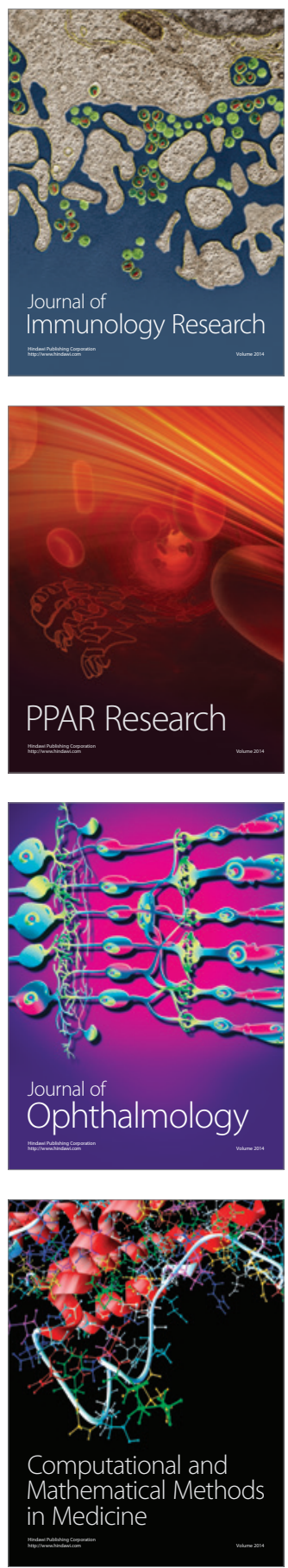

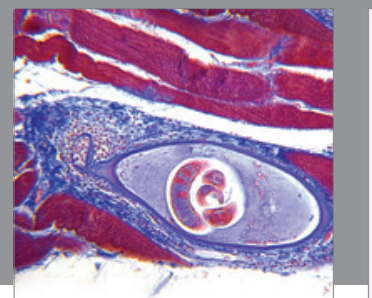

Gastroenterology

Research and Practice
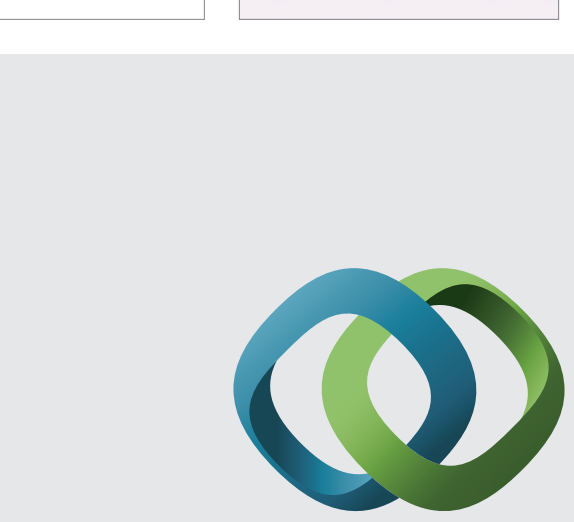

\section{Hindawi}

Submit your manuscripts at

http://www.hindawi.com
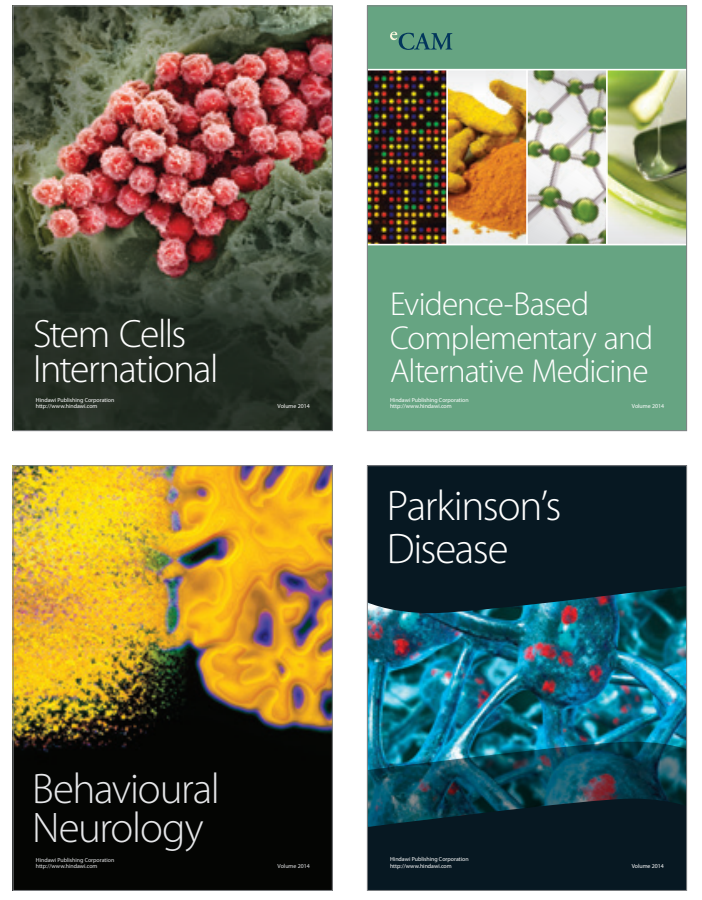
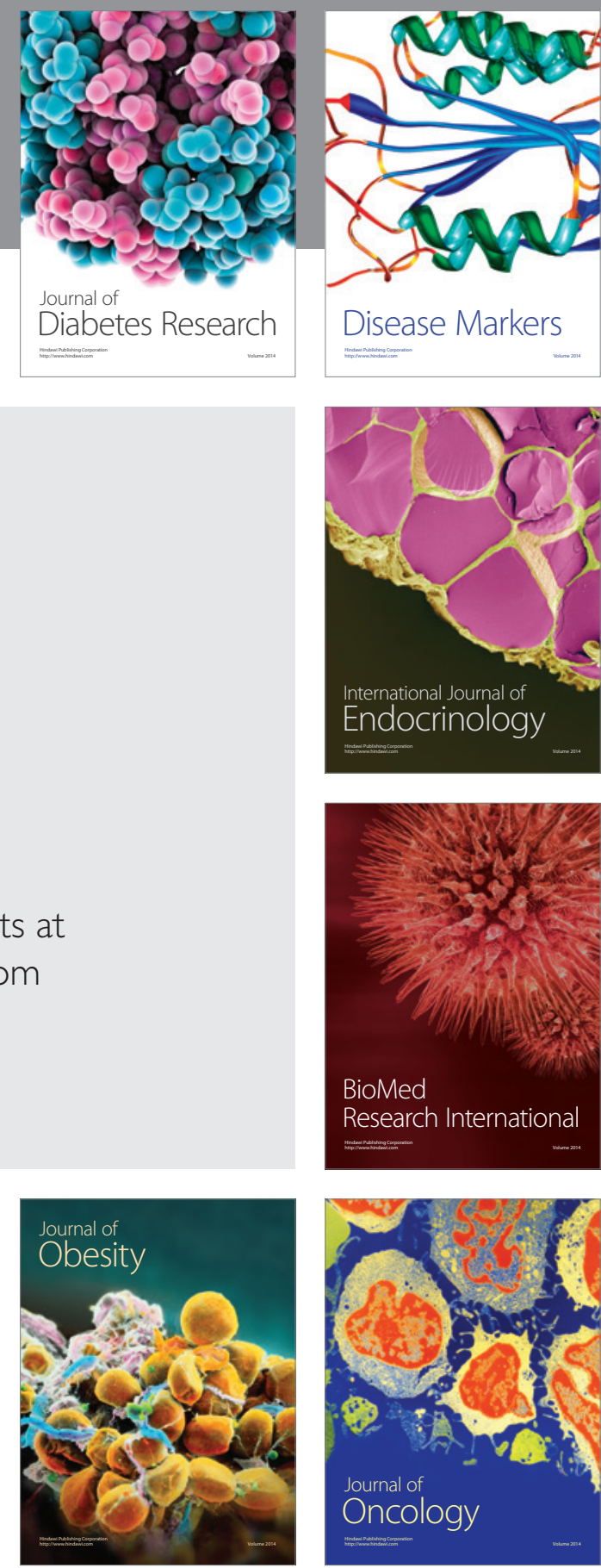

Disease Markers
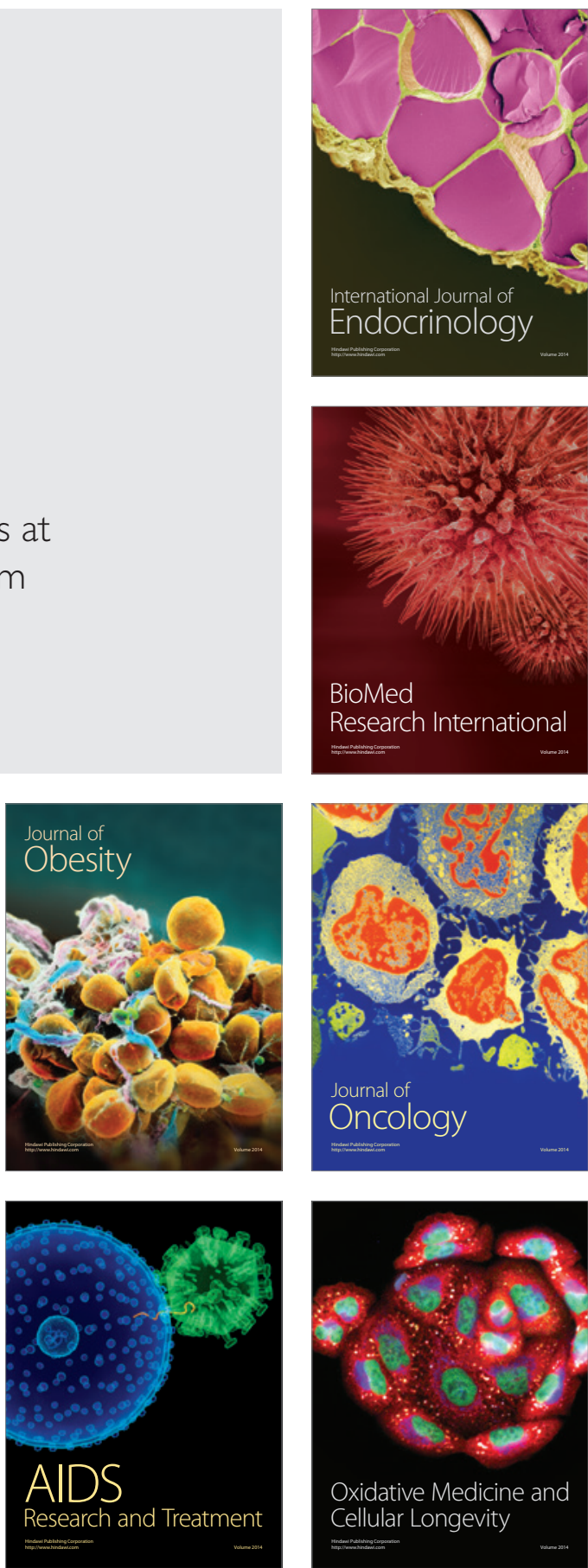